“ (C) 2019 IEEE. Personal use of this material is permitted. Permission from IEEE must be obtained for all other uses, in any current or future media, including

reprinting/republishing this material for advertising or promotional purposes, creating new collective works, for resale or redistribution to servers or lists, or reuse of any copyrighted component of this work in other works." 


\title{
Effect of Mechanical Resistance on Cognitive Conflict in Physical Human-Robot Collaboration
}

\author{
Stefano Aldini ${ }^{1}$, Ashlesha Akella ${ }^{2}$, Avinash K. Singh ${ }^{2}$, Yu-Kai Wang ${ }^{2}$, \\ Marc Carmichael ${ }^{1}$, Dikai Liu ${ }^{1}$ and Chin-Teng Lin ${ }^{2}$
}

\begin{abstract}
Physical Human-Robot Collaboration (pHRC) is about the interaction between one or more human operator(s) and one or more $\operatorname{robot}(\mathrm{s})$ in direct contact and voluntarily exchanging forces to accomplish a common task. In any pHRC, the intuitiveness of the interaction has always been a priority, so that the operator can comfortably and safely interact with the robot. So far, the intuitiveness has always been described in a qualitative way. In this paper, we suggest an objective way to evaluate intuitiveness, known as prediction error negativity (PEN) using electroencephalogram (EEG). PEN is defined as a negative deflection in event related potential (ERP) due to cognitive conflict, as a consequence of a mismatch between perception and reality. Experimental results showed that the forces exchanged between robot and human during pHRC modulate the amplitude of PEN, representing different levels of cognitive conflict. We also found that PEN amplitude significantly decreases $(p<0.05)$ when a mechanical resistance is being applied smoothly and more time in advance before an invisible obstacle, when compared to a scenario in which the resistance is applied abruptly before the obstacle. These results indicate that an earlier and smoother resistance reduces the conflict level. Consequently, this suggests that smoother changes in resistance make the interaction more intuitive.
\end{abstract}

\section{INTRODUCTION}

Human beings are highly adaptable and empowered by intellectual skills. On the other end, robots feature mechanical power and follow mathematical models that lead to a controlled behavior. When human and robot work together, the result is a confluent human-robot system that incorporates the strengths of both, but also add complexity and safety challenges. Collaborative robots are also referred as cobots [1].

There are several ways that robots and humans can interact with each other. Physical Human-Robot Interaction (pHRI) happens when a human and a robot are in physical contact. This pHRI can have a wide range of scenarios. The nature of the contact and the collaborative task can help define a more specific scope. This paper focuses on the physical Human-Robot Collaboration (pHRC) scenario in which a human operator and a robot are in contact (direct or through a rigid body) and voluntarily exchanging forces to accomplish a collaboration task. The human is fully or partially in control

\footnotetext{
${ }^{1}$ Stefano Aldini, Marc Carmichael and Dikai Liu are with the Centre for Autonomous Systems, Faculty of Engineering and Information Technology, University of Technology Sydney, 81 Broadway, Ultimo NSW 2010, Australia stefano.aldinieuts.edu.au

${ }^{2}$ Ashlesha Akella, Avinash K Singh, Yu-Kai Wang and Chin-Teng Lin are with the Centre for Artificial Intelligence, School of Software, Faculty of Engineering and Information Technology, University of Technology Sydney, 81 Broadway, Ultimo NSW 2010, Australia avinash.singheuts.edu.au
}

of the robot motions. It includes applications with the physical contact happening by means of haptic devices or objects that are being manipulated, and with continuous exchange of forces. Examples of pHRC with industrial robots include a cobot for material handling [2], a human-robot system for homokinetic joint assembly [3] and a wearable robot tested for lifting and holding tasks [4]. Another application of pHRC is rehabilitation robotics. Examples include a robot for hand rehabilitation [5], a lower-limb exoskeleton [6] and an exoskeleton to rehabilitate shoulder and elbow [7]. Figure 1 shows an example of an application involving pHRC, with an operator controlling a robot in a collaborative task. In this case, the operator is in physical contact with the handles mounted on the robot end-effector. The operator moves the robot through exchanged forces.

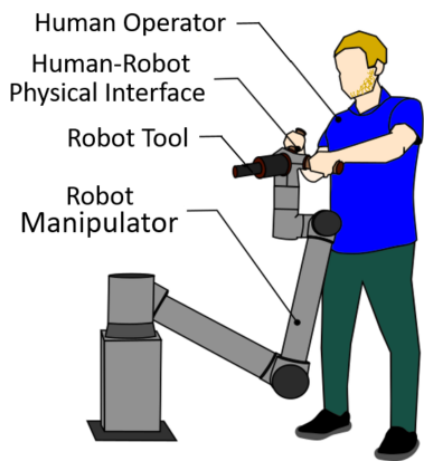

Fig. 1. An example of a pHRC application, with a human operator physically controlling a robot manipulator.

In pHRC applications, the human normally controls the robot through exchanged forces that are transformed into robot motions. Force-based controllers, such as impedance and admittance controls and their variants are normally used in this type of application [8]. The impedance, or admittance, of the human-robot system gives information about how forces are rendered into velocities. Often, the robot motion is also affected by other factors, such as collision and singularity avoidance and assistive strategies ([9], [10], [11], [12], [13]). Accounting for these factors means that motions of the robot are not solely dictated by the force interactions of the operator, but also by the result of the implemented strategies. Consequently, the human operator usually feels a variable mechanical resistance throughout the robot workspace. Usually, the resulting resistance is empirically adjusted based on factors such as smoothness and intuitiveness. 
The lack of a clear relationship between mechanical resistance and intuitiveness makes a rigorous mathematical approach impossible. In the literature, most of the pHRC studies claiming an intuitive interaction do not try to assess its intuitiveness. The few studies that addressed the intuitiveness, did it in a subjective way through surveys or verbal questions, such as [14].

Intuitiveness can be defined as the ability to know or understand things without rational thought. Bastick [15] describes it as a cognitive process based on previously perceived information, thus experience. In fact, intuition is based on the human internal models and the ability of the person to predict. Consequently, we can define that the intuitiveness of an operator controlling a robot is proportionally related to how easily they can predict its behavior. Experience and training usually translate in tasks feeling more natural and intuitive to the user. The level of intuitiveness of a robot should however be independent of the operator's experience.

Cognitive conflict is a phenomenon that can be the result of a mismatch between perception and reality. The human neuromotor control system uses internal models to continuously predict actions and outcomes of a task. Cognitive conflict happens when those internal models are proven wrong, and, consequently, when expectations are not met. This can be used as an indirect measure of intuitiveness. Cognitive conflict can be represented by prediction error negativity [16] detected in event related potential (ERP) [17] using electroencephalogram (EEG) [18].

In the next section an overview of related works is presented. Section III describes the approach used to detect and extrapolate the cognitive conflict during $\mathrm{pHRC}$ and gives insight of the robot control loop and how the mechanical resistance is modeled. Finally the experimental setup and results are presented and discussed.

\section{BACKGROUND}

The main challenges in pHRC are to ensure safety and to have an intuitive interaction that can take full advantage of the operator's skills [19]. There are several possible approaches to boost the interaction between human and robot. One of them aims to close the gap between the human and the robot by having a more natural flow of information. A robot is aware of the state of the human operator through its sensors. Biomedical devices that can measure physiological parameters are becoming more reliable, less expensive and user-friendly. Several research groups are looking into integrating those devices to have an estimation of the human state. During the interaction, galvanic skin responses can be used to measure engagement [20] and comfort [21]. Devices to measure the heart rate are more common and have been extensively used to measure the human state. Examples are Rani et al. [22], Villani et al. [23] and Jelinek et al. [24] that used it to estimate the psychological stress. The heart rate is however affected by many factors, physical and psychological. Combination of more than one biological signal are used by Kulíc and Croft [25], that used heart rate, facial muscle contraction and skin conductance responses, and Koenig et al. [26], that used heart rate, skin temperature and skin conductance. Biomedical signals in $\mathrm{pHRC}$ are mostly used to characterize the stress and comfort of the user.

Electroencephalographs (EEGs) are devices that measure the electrical activity from the brain, using electrodes placed on the scalp. The major advantages of EEG over other neuro-physiological measurement devices are its noninvasive nature, cost effectiveness, high temporal resolution and the possibility to work in active motion. EEG has been extensively used in cognitive science research [27] and for designing Brain Robot Interaction (BRI) systems [28]. In recent years, EEG has been proven robust in detecting specific features in the human state, such as prediction errors during ongoing actions. Although prediction error due to cognitive conflict has been investigated for a long time, only few works have focused on ongoing prediction error. The first case of research toward cognitive conflict was conducted by Falkenstein et al [29]. In a bimanual-choice reaction task, they found a feature in EEG signals known as errorrelated negativity (ERN). Following this work, several other researchers developed flanker tasks [30] to measure cognitive conflict in different scenarios. Most of these tasks are based on observational mismatch conditions on participants in a passive state with limited motor movement. Recently, studies that aimed to generate cognitive conflicts were conducted in scenarios which involved active motor movement [16] and found another EEG feature known as prediction error negativity (PEN). Since PEN is not self-generated, the frontal negativity is different from ERN and the resulting peak is different from the observational error that appears at approximately $300-400 \mathrm{~ms}$ after a stimulus. Such cognitive conflict for active motor movement are highly suitable for real-world applications like pHRC.

Recently Salazar-Gomez et al. [31] and Ehrlich and Cheng [32] used ERN during a human-robot interactive task. There was however no physical interaction and the user was supervising the task performed by a robot in the first case, and socially interacting in the second.

We propose that PEN can be used to adapt the robot behavior and support the user during the interaction, rather than using the brain activity to directly control the robot. To do so, it is important to understand how the physical interaction, in terms of forces, affects cognitive conflicts and, consequently, intuitiveness.

\section{EXPERIMENTAL METHOD}

In pHRC, the interaction between human and robot is mostly based on the force exchange and controls such as impedance- or admittance-based controllers. Mechanical resistance is often used as a way to guide the operator through the robot workspace, and to encourage or discourage some direction of motions. For example, a higher resistance can be used to guide the user away from undesirable configurations such as singularities and self-collisions. Since mechanical resistance is not visible, and thus hard to predict, it affects the intuitiveness of the human-robot interaction. 
In our approach, we use a human-robot system to perform a pHRC task. The operator will build an internal model of the task with the perceived information. Randomly, the user will experience a mechanical resistance opposing their motion. Since the mechanical resistance is invisible, this will prove wrong the human internal model previously created, causing a cognitive conflict. This setup allows the relationship between the mechanical resistance and the resulting PEN to be investigated.

\section{A. EEG preprocessing}

Raw EEG signals are filtered using a 1-Hz high-pass and a 50-Hz low-pass finite impulse response (FIR) filter. Subsequently, the data is downsampled to $250 \mathrm{~Hz}$ and independent component analysis (ICA) [33] is applied. Independent components (ICs) related to eye movement, muscle activity and other noise are rejected using artifact subspace reconstruction (ASR) [34] and automatic EEG artifact detection based on the joint use of spatial and temporal features (ADJUST) [35]. Epochs are extracted from $500 \mathrm{~ms}$ before the force stimulus is applied to the user to $1000 \mathrm{~ms}$ after the stimulus. In this paper the force stimulus is represented by the mechanical resistance.

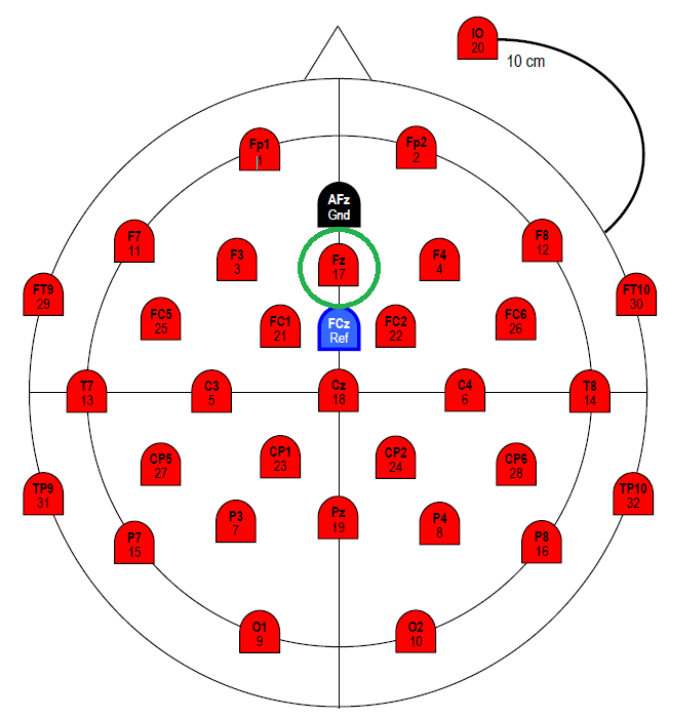

Fig. 2. A 32-channel EEG cap used for experiment where the green highlighted channel i.e. Fz has been used to extract PEN amplitude

\section{B. Identification of cognitive conflict}

Cognitive conflict is represented by PEN which is a negative deflection in ERP happening around $150-250 \mathrm{~ms}$ in the frontal area of brain. Therefore, we focus on ERP in $\mathrm{Fz}$ (see Figure 2), which is a mid-line frontal EEG channel. The PEN peak is found by calculating the average of the minimum peak happening in the time range of $150-250 \mathrm{~ms}$ with \pm 5 adjacent points in the extracted ERP.

\section{Control system}

An admittance control system is used to transform interaction forces into robot velocities. The scheme presented in
[36] is adapted for this specific application. The commands to the robot are in terms of joint velocities and are calculated using the inverse kinematic of the robot:

$$
\dot{q}=J^{-1}(q) \dot{x}_{d}
$$

With $\dot{q}$ being the joint velocities of the robot, $J$ being the Jacobian matrix and $\dot{x}_{d}$ the vector of desired velocities of the robot end-effector in the Cartesian space. Because the task uses only a small portion of the robot workspace, it was possible to limit the robot motions to areas in which the manipulator would always be in non-singular configurations, so that the Jacobian matrix is always invertible. Thus, the controller has no active singularity avoidance strategy, which would induce unintended mechanical resistance. The desired Cartesian velocities are calculated as follows:

$$
\dot{x}_{d}=A\left(f_{H}+f_{D}-f_{R}\right)
$$

With $A$ the matrix containing the admittance gains, $f_{H}$ the interaction forces exchanged with the operator, $f_{R}$ the force responsible of the mechanical resistance and $f_{D}$ a force that smoothen the velocities in the Cartesian space.

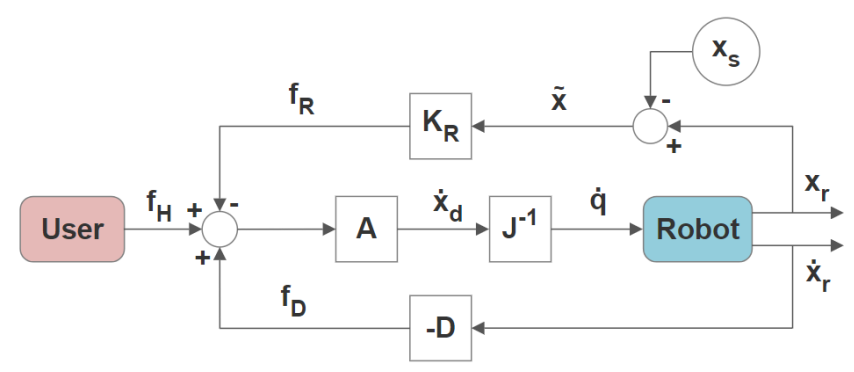

Fig. 3. The implemented admittance control.

In Figure 3 the block diagram of the implemented control system is shown.

The force $f_{D}$ is described by:

$$
f_{D}=-D \dot{x}_{r}=-D J \dot{q}
$$

With $\dot{x}_{r}$ being a vector containing the actual Cartesian velocities of the robot end-effector, calculated multiplying the Jacobian of the robot by the joint velocities. $D$ is a matrix of gains acting as a damper.

For this study, to reduce potential disturbances affecting the operator and its mental state, the motion is limited to one direction. The robot is free to move only along one axis of the Cartesian space.

Position in the other directions and in orientation is maintained using a basic $\mathrm{P}$ controller. If $i$ is the direction, and $i=1$ is the direction of motion, the overall control system can be summarized by:

$$
\left\{\begin{array}{l}
\dot{x}_{i}=A\left(f_{H}^{i}+f_{D}^{i}-f_{R}^{i}\right), \quad \text { if } \quad i=1 \\
\dot{x}_{i}=K_{P}\left(x_{d}^{i}-x_{r}^{i}\right), \quad \text { if } \quad 2 \leq i \leq 6
\end{array}\right.
$$

With $K_{P}=0.2$, expressed in $N / m$ for $i=2,3$ and $N / \mathrm{rad}$ for $i=4,5,6$, as the proportional gains for the 
position control. The desired Cartesian position of the $i$-th direction is $x_{d}^{i}$, and $x_{r}^{i}$ is the actual Cartesian position.

The force $f_{R}$, responsible of the mechanical resistance, is the result of Hooke's Law:

$$
f_{R}=K_{R} \tilde{x}
$$

The stiffness $K_{R}$ is multiplied by $\tilde{x}$ which is the difference between the position of the end-effector and a position where the mechanical resistance is supposed to start. The mechanical resistance is further described in Section IV.C.

\section{EXPERIMENTAL SETUP}

A collaborative robotic system called ANBOT, was used to perform the experiment. Figure 4 shows the ANBOT system. It features a UR10 arm by Universal Robots [37] and between the end-effector and the robotic arm, an ATI mini-45 is used to measure the interaction forces and torques. The operator is in physical contact with the robot through handle bars mounted on the robot end-effector, as showed in Figure 1. As mentioned above, only one direction of the robot arm is enabled for this experiment. In fact, the operator can move the robot only horizontally, parallel to the wall. The use of a 6-degrees-of-freedom arm is justified by the will of the authors to move this research to multi-dimentional tasks in the next future.

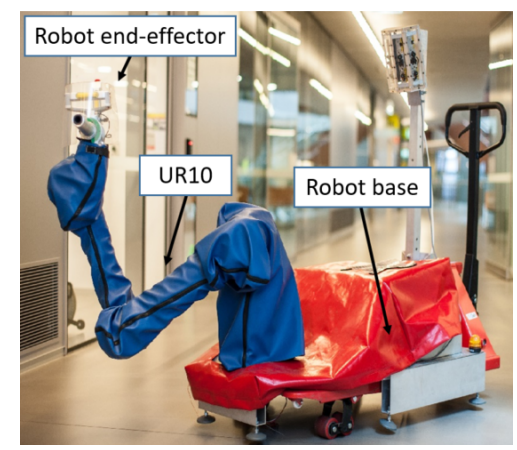

Fig. 4. The ANBOT system.

A 32-channel wireless EEG system (Brain Product GmbH, Germany) has been used. The placement of EEG system electrode was consistent with the 10-20 international system [38]. The contact impedance was maintained below $25 k \Omega$ with a sampling rate of $500 \mathrm{~Hz}$.

Data were collected from four healthy participants, 3 male and 1 female between 27 and 33 years of age. This experiment follows the procedure approved by the UTS Ethical Committee with approval number ETH15-0038.

Each participant performed 4 sets of 125 trials (500 trials in total), as detailed in Section IV-A. Two sets presented a resistance starting right before the target or the obstacle, while in the other two sets the resistance started earlier. The 4 sets were performed in random order.

Figure 5 shows one of the participants collaborating with the ANBOT to perform the experiment.

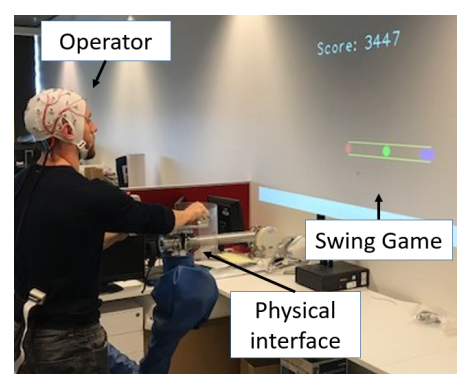

Fig. 5. A participant running the experiment while his brain waves are recorded.

\section{A. The Swing Game}

A game called the "Swing Game" was implemented to build a repetitive task and is shown in Figure 6. It is monoaxial, and as simple as possible to avoid giving too many inputs to the user and to keep them focused on the task.

The blue circle represents the point the nozzle of the robot is aiming at; from here on it will be referred as the cursor. Three possible targets are available: center, left and right. The current target, where the user is instructed to move the cursor, is green, while the others are red. In order to keep the user more engaged and to use both his hands, a blasting scenario was emulated: holding pressed only the back handle of the robot end-effector would move the robot and the cursor, while holding pressed the front handle would paint blue the area where the cursor is.

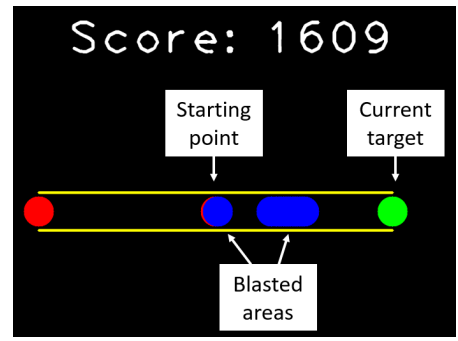

Fig. 6. The swing game. The red circles represent potential targets, the green circle is the current target while the blue areas is where the user has already blasted.

Because trials always start from the center, we will refer to this as the starting point. One of the other two targets is randomly turned from red to green. The user has 5 seconds to reach the target otherwise the trial is reset and the center target becomes green, to let the user know that they have to go back.

With a probability of $40 \%$ an invisible obstacle is placed halfway between the cursor and the target. This low probability was used to lessen the likelihood of operators creating an internal model of where the obstacle was likely to be encountered. This obstacle stops the motion of the robot and consequently of the cursor. Since this obstacle is not shown to the user, it causes a cognitive conflict: the operator will keep aiming to the green target, but, unexpectedly, will not be able to reach it. The robot will not move until the trial time is over even if the operator reaches the obstacle or the 
target.

The score displayed on screen is proportional to the blasted area and inversely proportional to the distance from the target when the time for the trial is over. It is only used to keep the user slightly engaged in the game.

\section{B. Mechanical resistance}

The mechanical resistance is calculated in the projected image space. Given Equation 5, the stiffness is set to $K_{R}=$ 0.5 and $x_{r}$ is transformed in $x_{p}$, the point of the image aimed by the nozzle of the ANBOT:

$$
\tilde{x}=x_{p}-x_{s}
$$

With $x_{s}$ being a point located before the target and the obstacle. In two sets of trials, to have a sudden resistance, $x_{s}$ is located $2.3 \mathrm{~cm}$ before the obstacle and the target. In the other two sets, to have a smoother resistance, starting further away from the obstacle and the target, $x_{s}$ is positioned $9.2 \mathrm{~cm}$ before the obstacle. $K_{R}$ and $x_{s}$ are set in a way to have a clear difference between the different conditions. This translates in the four conditions shown in Figure 7 and better explained in the next section.

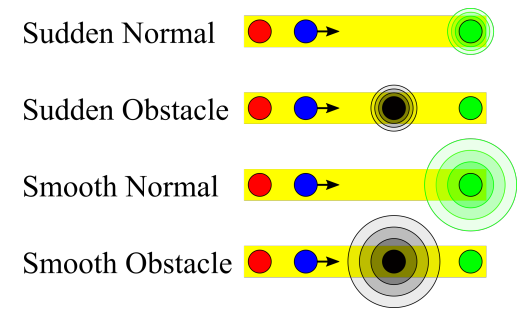

Fig. 7. The four conditions in the Swing Game.

\section{RESULTS AND DISCUSSION}

The total 2000 trials can be divided in four sets, given the different conditions (Figure 7):

1) 'Sudden Normal (Su-Nor)': sudden force, without obstacle;

2) 'Sudden Obstacle (Su-Ob)': sudden force, with obstacle;

3) 'Smooth Normal (Sm-Nor)': smooth force, without obstacle;

4) 'Smooth Obstacle (Sm-Ob)': smooth force, with obstacle.

For sake of clarity, Normal conditions refer to trials without an obstacle, while Obstacle conditions present the invisible obstacle. The words Sudden and Smooth are used to describe the used mechanical resistance. For example, a Sudden Obstacle ( $\mathrm{Su}-\mathrm{Ob})$ condition defines trials with a sudden mechanical resistance and an invisible obstacle.

The average of the interaction forces in the direction of motion for the four conditions are presented in Figure 8. These forces are measured by the load cell mounted on the robot. When a smooth resistance is applied the user takes more time to reach the target (or the obstacle) when

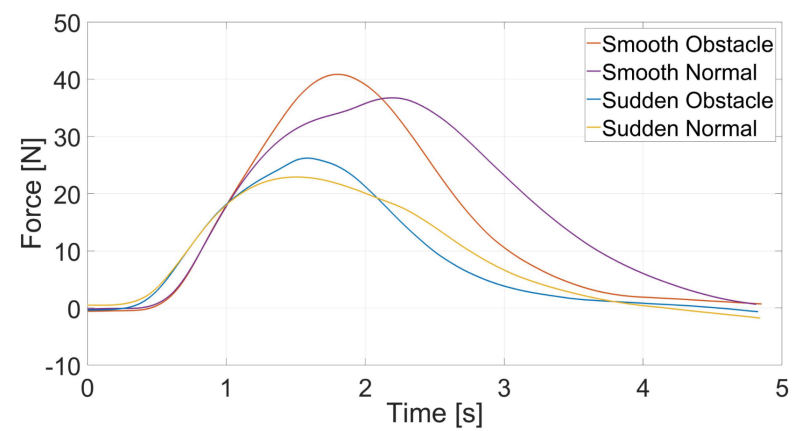

Fig. 8. Interaction forces measured by the force/torque sensor in the direction of motion for each condition. The curves are an average made on all the trials with the same conditions.

compared to sudden resistance conditions. The interaction force $f_{H}$ in (4) keeps increasing due to the resistance till the operator reaches the obstacle or the target. Forces are higher in case of smooth resistance due to the way $f_{R}$ is modeled (5). Since the Obstacle conditions present an invisible obstacle located halfway between the starting point and the target, the related curves present a peak slightly shifted on the left, when compared to the curves for Normal conditions.

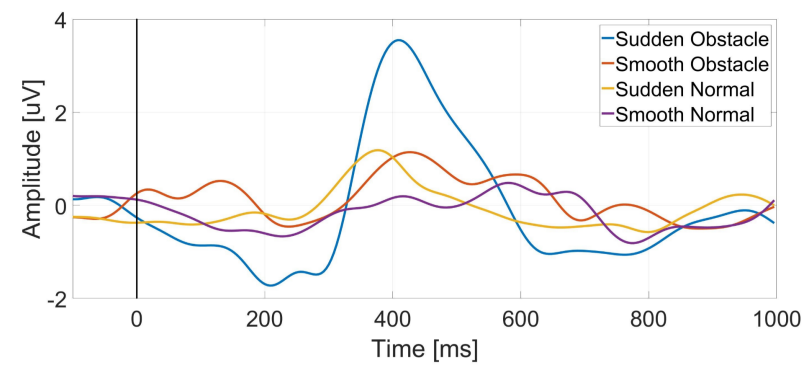

Fig. 9. Average of the ERP amplitude for each of the condition. The zero-time corresponds to when the user enters the area with a mechanical resistance.

Trials were grouped according to conditions and the ERP computed for each set. The average of the ERP is calculated using all the trials run by the different participants operating in the same condition. The result are shown in Figure 9. For the Sudden Obstacle condition it can be seen that there is a significant ( $p<0.05$ using paired-sample t-test) negative deflection around $200 \mathrm{~ms}$ followed by a positive peak at $400 \mathrm{~ms}$, known as P300 and related to memory processing. After a stimulus different from the expected one is received, a P300 is generated to update the internal models [39]. The ERP amplitudes of all the other conditions are comparable to the baseline, relative to the time range going from $-100 \mathrm{~ms}$ to $0 \mathrm{~ms}$. In this range the operator has not entered the area where the mechanical resistance is applied.

As shown in Figure 10, the ERP amplitudes of a single subject present a similar behaviour of the average, with a clear negative peak in case of an obstacle with sudden resistance.

We also investigated the PEN amplitude for all conditions for each participant to make sure cognitive conflict is hap- 


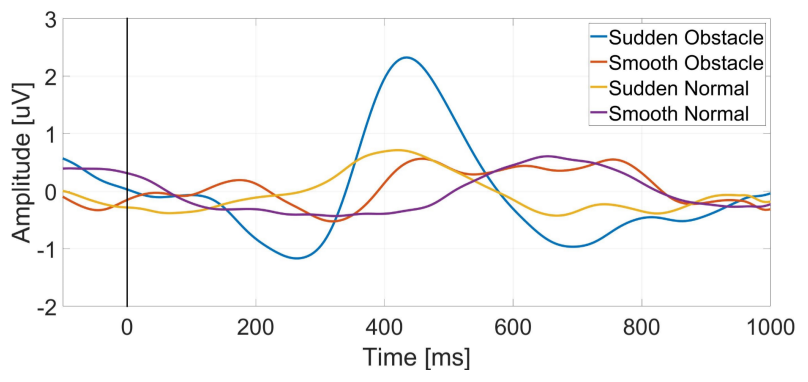

Fig. 10. ERP amplitude for each of the condition for a single participant. The zero-time corresponds to when the user enters the area with a mechanical resistance.

pening at an individual level. As it can be seen from Table I, for each participant, the PEN amplitude is significantly higher (more negative) for Sudden Obstacle condition when compared to Smooth Obstacle, Sudden Normal, and Smooth Normal. These results further strengthen the concept that PEN can be used to estimate the intuitiveness effectively and objectively.

TABLE I

AVERAGE PEN AMPLITUDE FOR EACH PARTICIPANT ORGANIZED BY CONDITION.

\begin{tabular}{|c|c|c|c|c|}
\hline \multirow{2}{*}{ Participant } & \multicolumn{4}{|c|}{ PEN Amplitude $(\mu$ V) } \\
\cline { 2 - 5 } & Su-Ob & Sm-Ob & Su-Nor & Sm-Nor \\
\hline S1 & -1.0786 & -0.0066 & -0.2839 & -0.6513 \\
\hline S2 & -1.4791 & -0.3895 & -0.4473 & -0.6795 \\
\hline S3 & -1.7164 & -1.0034 & -1.1080 & -0.4853 \\
\hline S4 & -1.1466 & -0.2015 & -0.2420 & -0.4035 \\
\hline
\end{tabular}

Figure 11 shows the difference in amplitude of the ERPs obtained in Normal conditions (without obstacle) from the ones with the obstacle. The effect of the PEN around $250 \mathrm{~ms}$ becomes more prominent and significant $(p<0.05)$ in the case of sudden resistance. In case of smooth resistance, starting further away from the obstacle, the effect of the PEN is negligible.

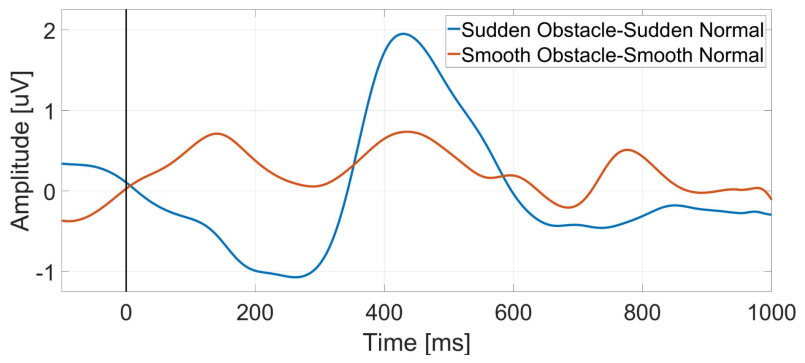

Fig. 11. Difference of the ERP amplitude between the conditions featuring an obstacle and the ones without. The zero-time corresponds to when the user enters the area with a mechanical resistance.

The results presented in Figure 9 and Figure 11 suggest that the mechanical resistance modulates the PEN. In Smooth Obstacle conditions, so with a wider, smoother resistance around the invisible obstacle, the operator presented less conflict in terms of PEN amplitude (less negative). On the contrary, suddenly applied mechanical resistance results in a more negative PEN (see Figure 12), thus in a greater cognitive conflict.

Therefore, suddenly providing the operator with a sharp resistance or abruptly interrupting the motion of a robot during a pHRC task, creates a cognitive conflict. Since we hypothesize cognitive conflict being a tool for estimating how intuitive is the interaction, we conjecture that applying sudden resistance to the operator's motion is counter-intuitive.

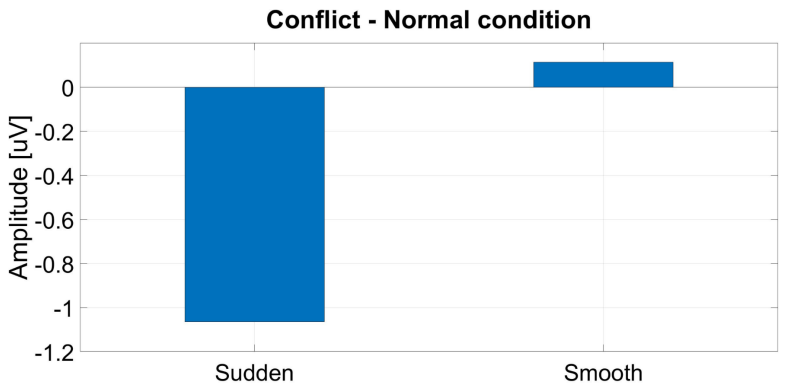

Fig. 12. ERP amplitude in case of sudden resistance and smooth resistance.

Consequently, results suggest that applying wider and smoother resistive forces around undesired areas of the workspace might reduce the level of conflict. This is probably due to the fact that the operator is able to better predict the change of conditions in the workspace is the robots starts resisting the motion sooner and more gently.

\section{CONCLUSIONS}

Achieving an intuitive interaction is the goal of most systems in pHRC. Intuitiveness is however used as a subjective feature of systems. Given that is possible to identify PEN from EEG recordings, it is possible to quantify the mismatch between predictions and reality. This can be used as an estimation of intuitiveness. Since the interaction in pHRC happens mainly through forces exchanged between the operator and the robot, the behavior of these forces greatly affects the whole interaction.

An experiment was designed to prove that the way mechanical resistance is designed in a robot workspace modulates PEN. Results prove that a wider, smoother resistance around an undesired area of the workspace helps the operator predicting upcoming changes in the interaction. This may be interpreted as a more intuitive interaction.

This opens possibilities for improving of control systems. Having an on-line estimation of the intuitiveness can lead to control systems that adjust the impedance of the robot to achieve a given level of that intuitiveness. Also, having an objective measure of system intuitiveness can be used to guide the development of improved pHRC paradigms.

\section{ACKNOWLEDGMENT}

This study is supported in part by the Australian Research Council (ARC) Linkage Project [LP140100950] and the ARC Discovery Project Grant [DP180100670]. 


\section{REFERENCES}

[1] J. E. Colgate, W. Wannasuphoprasit, and M. A. Peshkin, "Cobots: Robots for collaboration with human operators," 1996.

[2] E. Gambao, M. Hernando, and D. Surdilovic, "A new generation of collaborative robots for material handling," Gerontechnology, 2012.

[3] A. Cherubini, R. Passama, A. Crosnier, A. Lasnier, and P. Fraisse, "Collaborative manufacturing with physical human-robot interaction," Robotics and Computer-Integrated Manufacturing, 2016.

[4] T. Aida, H. Nozaki, and H. Kobayashi, "Development of muscle suit and application to factory laborers," in 2009 IEEE International Conference on Mechatronics and Automation, ICMA 2009, 2009.

[5] L. Masia, H. I. Krebs, P. Cappa, and N. Hogan, "Whole-arm rehabilitation following stroke: Hand module," in Proceedings of the First IEEE/RAS-EMBS International Conference on Biomedical Robotics and Biomechatronics, 2006, BioRob 2006, 2006.

[6] H. Yu, S. Huang, G. Chen, Y. Pan, and Z. Guo, "Human-Robot Interaction Control of Rehabilitation Robots with Series Elastic Actuators," IEEE Transactions on Robotics, 2015.

[7] M. G. Carmichael and D. K. Liu, "Human biomechanical model based optimal design of assistive shoulder exoskeleton," in Springer Tracts in Advanced Robotics, 2015.

[8] F. Rezazadegan, J. Geng, M. Ghirardi, G. Menga, S. Murè, G. Camuncoli, and M. Demichela, "Risked-based Design for the Physical Human-Robot Interaction (pHRI): an Overview," Chemical Engineering Transactions, vol. 43, pp. 1249-1254, 2015.

[9] M. G. Carmichael, D. Liu, and K. J. Waldron, "A framework for singularity-robust manipulator control during physical human-robot interaction," The International Journal of Robotics Research, 2017.

[10] D. Oetomo and M. H. Ang, "Singularity robust algorithm in serial manipulators," Robotics and Computer-Integrated Manufacturing, 2009.

[11] A. S. Deo and I. D. Walker, "Overview of damped least-squares methods for inverse kinematics of robot manipulators," Journal of Intelligent \& Robotic Systems, 1995.

[12] B. Navarro, A. Fonte, P. Fraisse, G. Poisson, B. Navarro, A. Fonte, P. Fraisse, G. Poisson, A. Cherubini, and B. Navarro, "OpenPHRI : an open-source library for safe physical human-robot interaction," Hal, 2017.

[13] A. De Luca and F. Flacco, "Integrated control for pHRI: Collision avoidance, detection, reaction and collaboration," in Proceedings of the IEEE RAS and EMBS International Conference on Biomedical Robotics and Biomechatronics, 2012.

[14] F. Ficuciello, L. Villani, and B. Siciliano, "Variable Impedance Control of Redundant Manipulators for Intuitive Human-Robot Physical Interaction," IEEE Transactions on Robotics, 2015.

[15] T. Bastick, Intuition: How We Think and Act. Toronto: John Wiley \& Sons Inc, 1982.

[16] A. K. Singh, H. T. Chen, Y. F. Cheng, J. T. King, L. W. Ko, K. Gramann, and C. T. Lin, "Visual Appearance Modulates Prediction Error in Virtual Reality," IEEE Access, 2018.

[17] P. L. Nunez and R. Srinivasan, Electric Fields of the Brain: The neurophysics of EEG. 2009.

[18] S. J. Luck, "Event-Related Potentials," APA Handbook of Research Methods in Psychology, 2005.

[19] V. Villani, F. Pini, F. Leali, and C. Secchi, "Survey on human-robot collaboration in industrial settings: Safety, intuitive interfaces and applications," 2018.

[20] E. Mower, D. J. Feil-Seifer, M. J. Matarić, and S. Narayanan, "Investigating implicit cues for user state estimation in human-robot interaction using physiological measurements," in Proceedings - IEEE International Workshop on Robot and Human Interactive Communication, 2007.
[21] E. Meisner, V. Isler, and J. Trinkle, "Controller design for human-robot interaction," Autonomous Robots, 2008.

[22] P. Rani, J. Sims, R. Brackin, and N. Sarkar, "Online stress detection using psychophysiological signals for implicit human-robot cooperation," Robotica, 2002.

[23] V. Villani, L. Sabattini, C. Secchi, and C. Fantuzzi, "Natural interaction based on affective robotics for multi-robot systems," in 2017 International Symposium on Multi-Robot and Multi-Agent Systems, MRS 2017, 2018.

[24] H. F. Jelinek, K. G. August, M. H. Imam, A. H. Khandoker, A. Koenig, and R. Riener, "Cortical response to psycho-physiological changes in auto-adaptive robot assisted gait training," in Proceedings of the Annual International Conference of the IEEE Engineering in Medicine and Biology Society, EMBS, 2011.

[25] D. Kulíc and E. Croft, "Affective state estimation for human-robot interaction," in IEEE Transactions on Robotics, 2007.

[26] A. Koenig, X. Omlin, L. Zimmerli, M. Sapa, C. Krewer, M. Bolliger, F. Mller, and R. Riener, "Psychological state estimation from physiological recordings during robot-assisted gait rehabilitation," The Journal of Rehabilitation Research and Development, 2011.

[27] J. Friedenberg and G. Silverman, Cognitive Science: An Introduction to the Study of Mind. 2005.

[28] X. Mao, M. Li, W. Li, L. Niu, B. Xian, M. Zeng, and G. Chen, "Progress in EEG-based brain robot interaction systems," Computational Intelligence and Neuroscience, 2017.

[29] M. Falkenstein, J. Hohnsbein, J. Hoormann, and L. Blanke, "Effects of crossmodal divided attention on late ERP components. II. Error processing in choice reaction tasks," Electroencephalography and Clinical Neurophysiology, 1991.

[30] B. Kopp, F. Rist, and U. Mattler, "N200 in the flanker task as a neurobehavioral tool for investigating executive control," Psychophysiology, 1996

[31] A. F. Salazar-Gomez, J. Delpreto, S. Gil, F. H. Guenther, and D. Rus, "Correcting robot mistakes in real time using EEG signals," in Proceedings - IEEE International Conference on Robotics and Automation, 2017.

[32] S. K. Ehrlich and G. Cheng, "Human-agent co-adaptation using errorrelated potentials," Journal of neural engineering, 2018.

[33] S. Makeig, A. J. Bell., T.-P. Jung, and T. J. Sejnowski, "Independent Component Analysis of Electroencephalographic Data," Advances in Neural Information Processing Systems, 1996.

[34] C. Y. Chang, S. H. Hsu, L. Pion-Tonachini, and T. P. Jung, "Evaluation of Artifact Subspace Reconstruction for Automatic EEG Artifact Removal," Conference proceedings of the Annual International Conference of the IEEE Engineering in Medicine and Biology Society, 2018.

[35] A. Mognon, J. Jovicich, L. Bruzzone, and M. Buiatti, "ADJUST: An automatic EEG artifact detector based on the joint use of spatial and temporal features," Psychophysiology, 2011.

[36] M. G. Carmichael and D. Liu, "Admittance control scheme for implementing model-based assistance-as-needed on a robot," in Proceedings of the Annual International Conference of the IEEE Engineering in Medicine and Biology Society, EMBS, 2013.

[37] Universal Robots, "UR10 Collaborative industrial robotic arm - Payload up to $10 \mathrm{~kg}$." https://www.universal-robots.com/products/ur10robot/.

[38] G. E. Chatrian, E. Lettich, and P. L. Nelson, "Ten percent electrode system for topographic studies of spontaneous and evoked EEG activity," American Journal Of EEG Technology, 1985.

[39] J. Polich, "Updating P300: An integrative theory of P3a and P3b,' Clinical Neurophysiology, 2007. 